\title{
Changes in cortical slow wave activity in healthy aging
}

\author{
Vera Maria Leirer - Christian Wienbruch • \\ Stephan Kolassa • Winfried Schlee - Thomas Elbert • \\ Iris-Tatjana Kolassa
}

\begin{abstract}
A number of studies have demonstrated enhanced slow wave activity associated with pathological brain function e.g. in stroke patients, schizophrenia, depression, Morbus Alzheimer, and post-traumatic stress disorder. However, the association between slow wave activity and healthy aging has remained largely unexplored. This study examined whether the frequency at which focal generators of delta waves appear in the healthy cerebral cortex changes with age and whether this measure relates to cognitive performance. We investigated 53 healthy individuals aged 18 to 89 years and assessed MEG during a resting condition. Generators of focal magnetic slow waves were localized. Results showed a significant influence of age: dipole density decreases with increasing age. The relationship between cognitive performance and delta dipole density was not significant. The results suggest that in healthy aging slow waves decrease with aging and emphasize the importance of age-matched control groups for further studies.
\end{abstract}

V. M. Leirer $\cdot$ C. Wienbruch $\cdot$ T. Elbert $\cdot$ I.-T. Kolassa Clinical Psychology \& Neuropsychology, University of Konstanz, Universitätsstr. 10

78457 Konstanz, Germany

\section{S. Kolassa}

Research \& Innovation, SAF Simulation,

Analysis \& Forecasting AG,

Bahnstr. 1,

8274 Tägerwilen, Switzerland

W. Schlee $\cdot$ I.-T. Kolassa ( $\)$

Clinical \& Biological Psychology,

Institute of Psychology \& Education, University of Ulm,

Albert-Einstein-Allee 47,

89069 Ulm, Germany

e-mail: Iris.Kolassa@uni-ulm.de

I.-T. Kolassa

Zukunftskolleg, University of Konstanz,

Box X 916, 78457 Konstanz, Germany
Increased appearance of slow waves as a marker for pathological stages can only be detected in relation to a control group of the same age.

Keywords Magnetoencephalography - Delta slow waves . Magnetic source imaging · Healthy aging · Cognitive performance

\section{Introduction}

Cognitive functions decline on average during aging, although substantial individual differences exist in onset, rate and scope (e.g. Backman et al. 2006; Colsher \& Wallace 1991; Corral et al. 2006; Evans et al. 1993; Wilson et al. 1999). For example, attention, short-term storage, visuomotor abilities, verbal memory and visual memory decline with increasing age (Corral et al. 2006). Furthermore, a study by Lindenberger and Reischies (1999) reported a decline of the five intellectual abilities: reasoning, memory, perceptual speed, knowledge and fluency. Different age trends for different cognitive variables were found by Salthouse 2006 (Salthouse 2006): performance in episodic memory, perceptual reasoning, perceptual speed and word knowledge starts to decline when people are in their 20 s or 30 s with a further and faster decline at around age 50. In contrast, an increase in performance is found for tasks with large knowledge components until the age of about 50 with a gradual decline afterwards.

At the brain level, studies on structural changes have demonstrated a decline in total (e.g. Giedd et al. 1999) and in regional brain volume, e.g. in the cingulate sulci, hippocampus, insula, caudate, cerebellum and the entorhinal cortices (e.g. Good et al. 2001; Raz et al. 2005), loss of synaptic connections (Terry \& Katzman 2001), atrophy of gray and 
white matter (Bartzokis 2004; Galluzzi et al. 2008). However, amyloid deposition has been detected even in non-demented older subjects (e.g. Aizenstein et al. 2008; Mintun et al. 2006; Pike et al. 2007). Morphological changes are therefore not sufficient for the prediction of cognitive decline.

Slow waves in the delta frequency range can be detected by electroencephalography (EEG) as well as by magnetoencephalography (MEG). However, since MEG allows the separation of focally generated slow waves from those with widespread sources, MEG appears better suited for the identification of focal slow wave activity than EEG (Wienbruch 2007). An equivalent current dipole model provides an excellent approximation to localize such focal assemblies of active pyramidal cells (Elbert 1998). Accordingly, in abnormal slow wave activity mapping (ASWAM) (Wienbruch 2007), generators of focal magnetic slow waves in the $1-4 \mathrm{~Hz}$ (delta) frequency range are localized by employing a single moving dipole model and by calculating dipole densities in anatomically defined cortical regions (frontal, central, temporal and parieto-occipital).

High densities of focal generators of such slow wave activity have been found to be related to brain pathology or dysfunctional brain tissue as a result of metabolism and blood flow changes after an insult (e.g. Baayen et al. 2003; de Jongh et al. 2001; Niedermeyer \& Lopes da Silva 1987; Vieth et al. 1996) or deafferentation of neuronal networks cut off from major input sources (e.g. Amzica \& Steriade 1997; Gloor et al. 1977). For example, brain lesions are frequently accompanied by slow waves in deafferented regions after stroke (Meinzer et al. 2004) or in circumscribed regions around a tumor (Baayen et al. 2003; de Jongh et al. 2001; Vieth et al. 1996). Furthermore, even in the absence of structurally obvious lesions, focal slow wave generators are also abnormally distributed in psychiatric diseases such as depression, schizophrenia and post-traumatic stress disorder (Fehr et al. 2001, 2003; Fernández et al. 2005; Kolassa et al. 2007; Rockstroh et al. 2007; Wienbruch et al. 2003).

Focal generators of slow wave activity have also been reported to play a significant role in the evaluation of pathological aging processes: In Alzheimer's disease (AD), clear focal temporoparietal slow wave activity was revealed, which was furthermore predictive of the cognitive status of the patients (Fernández et al. 2002) and linked to the rate of atrophy in the temporal lobe (Fernández et al. 2003). Additionally, left-parietal slow wave generator (delta dipoles) density was shown to be an effective predictor for mild cognitive impairment (MCI) to Alzheimer's disease (AD) conversion (Fernández et al. 2006) with an increasing risk of conversion to $\mathrm{AD}$ in those $\mathrm{MCI}$ patients with high left parietal delta dipole density.

However, there is also evidence that delta slow wave activity is not pathological per se: Meinzer and colleagues
(2004), for example demonstrated that abnormally elevated delta activity can sometimes be related to functionally favorable outcomes in patients with chronic aphasia following strokes after intense speech and language therapy.

So far, alterations in slow wave density have only been shown in pathological aging, but the distribution of slow waves during the normal, non-pathological aging process remains to be explored. To our knowledge, only Wienbruch (2007) investigated age effects in delta dipole density in healthy individuals. In this sample, only a small correlation between age and slow wave activity was found which led the author to conclude that age per se may not account for an enhancement of slow wave activity often seen in subjects with mild cognitive decline or dementia. However, this study did not assess cognitive performance and no individuals older than 66 years were included, i.e., neither changes during the relevant periods of mental aging nor association with cognitive performance were assessed.

The present study aimed at exploring age-related alterations in the distribution of generators of focal magnetic slow waves and associations between cognitive performance and sources of low-frequency activity in the brain using magnetic source imaging. For this purpose, we performed the above mentioned abnormal slow wave activity mapping (ASWAM) method that has been shown to be a valid tool in the vicinity of the detection of pathological attributed slow wave activity (Wienbruch 2007).

\section{Methods}

\section{Participants}

Fifty-three right-handed, healthy subjects (23 males and 30 females) ranging from 18 to 89 years $(M=53.06$ years, $S D=$ 20.07) participated in this study. Age was equally distributed with respect to gender. Their mean education was 15.11 years (ranging from 10 to 22 years). Participants were recruited by advertisement in the local newspaper and radio station as well as by notifications posted in and around Konstanz (campus, residential homes for the elderly, senior citizen centers, sports clubs etc.) and. They were paid $30 €$ for participation. Exclusion criteria were acute as well as a history of psychiatric diseases, a history of psychopharmacological medication, left-handedness, metal objects in the body as well as a history of severe head injuries or neurological problems such as epilepsy, strokes, brain tumors. The ethics committee of the University of Konstanz approved this study.

Procedures

Upon arrival at the laboratory, participants were familiarized with the MEG chamber (a magnetically shielded room; 
MSR; Vakuumschmelze Hanau), and study procedures and goals were clarified. All participants provided written informed consent. Afterwards subjects were screened with the MINI International Neuropsychiatric Interview (Ackenheil et al. 1999) to assure that participants who were included in the study did not suffer from psychiatric problems. Subsequently, demographic data were assessed and handedness was determined using the Edinburgh Inventory (Oldfield 1971). Furthermore, cognitive abilities were assessed with the CERAD-NP-plus test battery (Morris et al. 1988) with the subtests Verbal Fluency (VF=sum score of semantic and phonemic fluency), Word List Learning (WLL), Word List Delayed Recall (WLDR), Word List Recognition (WLR), Figure Recall (FR), Trail Making Test A and B (TMT-A/B). Additionally, the Digit Symbol, the Digit Span and the Mosaic subtests of the German version of the Wechsler Adult Intelligence Scale (HAWIE-R; Tewes 1991) as well as the Benton Visual Retention Test - revised form (Steck 2005) were conducted.

\section{MEG data acquisition}

After the neuropsychological assessment, MEG recordings were obtained during a $5 \mathrm{~min}$ resting period. For artifact rejection, we recorded the electro-occulogram (EOG) using a bipolar montage where electrodes were attached near the left and right outer canthus (horizontal EOG) and below and above the right eye (vertical EOG). For recording of the electrocardiogram (ECG), two additional electrodes were attached at the left lower forearm and the right collarbone. Subsequently, participants were seated in the MSR and their index points and head shapes were digitized with a Polhemus 3 Space Fasttrack (Polhemus, Colchester, VT, USA). The nasion point, an anatomical landmark, and the left and right ear canal points served as index points and were used to define a right-handed coordinate system, called headframe coordinate system; the $x$-axis points to the front, the $y$-axis to the left and the $z$-axis to the top of the head. The subjects' headsize was measured using three parameters: ANTERIOR, which is the $x$-coordinate of nasion point; LEFTRIGHT, which is the difference of the $y$-coordinates of the left and right ear canal points; SUPERIOR, which is the maximum of the $z$-coordinate of all digitized head shape points. The subjects' head position relative to the pickup coils of the sensor was estimated before and after the measurement.

During MEG measurement subjects were lying supine in a comfortable position and were instructed to stay awake and not engage in any specific mental activity. They were further asked to fixate a mark on the ceiling of the magnetically shielded room and to avoid eye as well as body movements throughout the recording. A video camera installed inside the chamber allowed monitoring subjects' behavior and ensured compliance throughout the experiment.

MEG was recorded continuously and digitized at a rate of $678.17 \mathrm{~Hz}$ using a 148-channel whole head magnetometer (MAGNESTM, 2500 WH, 4D Neuroimaging, San Diego, USA). A real band-pass filter of $0.1-200 \mathrm{~Hz}$ was used for data acquisition. EOG and ECG were recorded with a SynAmps amplifier (Neuroscan ${ }^{\circledR}$ ) using $\mathrm{Ag} / \mathrm{AgCl}$ electrodes.

\section{Data analysis}

Slow wave generators were identified in a semi-automated procedure. The main steps were application of noise reduction, decimation, digital band pass filters and magnetic source imaging. Following noise reduction, data were screened for artifacts (e.g. eye blinks or muscle activity) by visual inspection and time periods without artifacts were selected. Data were then decimated by a factor 16 (anti-alias filters were applied automatically in the same processing step) and digitally filtered for the delta $(1.5-4.0 \mathrm{~Hz})$ frequency band using a zero phase shift digital band pass filter (Butterworth filter of order 4).

Single equivalent dipoles were fitted for each time point in the selected artifact-free segments. The selection of specific dipole solutions had to meet the criteria Goodness of Fit ( $\mathrm{GoF}>0.90$, to ensure statistical significance of the source model) and Dipole moment (Q 10-100 nAM, which may require that at least $0.1-1 \mathrm{~cm}^{2}$ of cortex is activated). Dipoles with an inferior-superior coordinate (z) below $0.0 \mathrm{~cm}$ and dipoles within $3 \mathrm{~cm}$ around the origin (i.e., near the center of the head) were excluded. Dipole density was estimated within a volume defined as a cube divided into voxels with a size of $2 \times 2 \times 2 \mathrm{~cm}^{3}$. The cube is determined from an ACPC (anterior commissural (AC) posterior commissural (PC)) based coordinate system, which, in turn, results from the individual headframe coordinate system, defined by the nasion, the left and the right ear channel). Each source volume (cube) comprises 1331 voxels. Each equivalent dipole is assigned to one voxel of the source volume, and the number of dipoles within a voxel is counted over time. As artifact-free time periods varied between subjects, dipole density was normalized by the subjects' individual number of artifactfree time points.

The normalized dipole density was calculated for each subject within each voxel of the source volume. Then the logarithm of the normalized dipole density was calculated in order to obtain a normal distribution across subjects. For the analysis of the abnormal slow wave activity (ASWA) distribution, dipole density was determined within anatomically defined brain areas, which were specified for temporal, frontal, central and parietal areas of the brain 
within each hemisphere following the classification of the anatomical atlas provided with MRICRO's AAL (http://www.sph.sc.edu/comd/rorden/mricro.html). Within these eight regions dipole density was estimated as the average of the voxel-based dipole density. (For a more detailed description of data analysis see Wienbruch (2007))

Statistical analysis

For data analysis, linear mixed effects models were implemented using R 2.8.1 (R Development Core Team 2008). In all mixed models, each subject had a random intercept whereas all other factors were fixed effects. AIC (Akaike 1973) was lowest for the following model equation: hemisphere $\times$ region + age + neuropsychological test-for instance, it was lower by 70 than the AIC for a model hemisphere $\times$ region $\times$ age + neuropsychological test, which would have allowed for different age effects in each region and hemisphere, which provides overwhelming evidence that the simpler model is a better approximation to the data generating process from both an information-theoretic and a Bayesian perspective (Burnham \& Anderson 2002). This model was analyzed for each neuropsychological test separately. To correct for multiple comparisons, the stepwise Holm method was applied.

For assessing age effects on test results (cognitive capacity), linear regressions were conducted using SPSS (SPSS Statistics 17.0) with the constant variable age and dependent variables test results.

\section{Results}

Statistical analysis revealed a significant negative effect between age and slow wave activity (Fig. 1). A medium effect size (partial eta $^{2}=.14$ ) was detected for age in all statistical models (see Table 1). Regardless of which neuropsychological covariate was used in the respective model, the effect of age always became highly significant. Depending on the neuropsychological covariate included in the model, the unstandardized estimated regression coefficient for age was between -0.019 and -0.013 . No significant effects for hemisphere, region or gender were found.

Regarding the relationship between cognitive performance and abnormal slow wave activity, only one significant association was observed (digit span task $F(1,50)=4.37 ; p=.04$ before Holm's stepwise correction).

Table 1 Relationships between the fixed effects neuropsychological tests and age and abnormal slow wave activity (model equation: Hemisphere $\times$ Region + age + neuropsychological tests). No significant effects for hemisphere and region were observed

\begin{tabular}{|c|c|c|c|c|}
\hline & $F$ (neuropsychological test) & $p$ (neuropsychological test) & $F$ (age) & $p$ (age) \\
\hline Digit Symbol & $F(1,49)=.007$ & .93 & $F(1,49)=11.74$ & $.001^{*}$ \\
\hline Digit Span & $F(1,50)=4.37$ & $.042 *$ & $F(1,50)=10.83$ & $.002 *$ \\
\hline Mosaic Test & $F(1,50)=.002$ & .97 & $F(1,50)=9.96$ & $.003^{*}$ \\
\hline Benton-corr & $F(1,49)=.288$ & .60 & $F(1,49)=11.81$ & $.001 *$ \\
\hline Boston Naming Test & $F(1,50)=1.66$ & .20 & $F(1,50)=10.29$ & $.002 *$ \\
\hline MMSE & $F(1,50)=3.26$ & .08 & $F(1,50)=10.61$ & $.002 *$ \\
\hline WLL & $F(1,50)=.06$ & .81 & $F(1,50)=9.97$ & $.003^{*}$ \\
\hline WLDR & $F(1,50)=.13$ & .73 & $F(1,50)=9.99$ & $.003^{*}$ \\
\hline WLR & $F(1,50)=.75$ & .39 & $F(1,50)=10.11$ & $.003 *$ \\
\hline FD & $F(1,50)=.02$ & .90 & $F(1,50)=9.96$ & $.003^{*}$ \\
\hline FR & $F(1,50)=.42$ & .52 & $F(1,50)=10.04$ & $.003 *$ \\
\hline VF & $F(1,50)=.02$ & .90 & $F(1,50)=9.96$ & $.003^{*}$ \\
\hline TMT-A & $F(1,50)=1.23$ & .27 & $F(1,50)=10.20$ & $.003^{*}$ \\
\hline TMT-B & $F(1,50)=.14$ & .71 & $F(1,50)=9.99$ & $.003^{*}$ \\
\hline
\end{tabular}

Digit Symbol $=$ Digit Symbol subtest of the German version of the Wechsler Adult Intelligence Scale; Mosaic Test $=$ Mosaic test subtest of the German version of the Wechsler Adult Intelligence Scale; Digit Span = Digit Span subtest of the German version of the Wechsler Adult Intelligence Scale; Benton-corr = correct answers of the Benton Visual Retention Test (revised form); MMSE = Mini Mental State Examination; WLL = Subtest Word List-Learning of the German version of the CERAD-NP-Plus test battery; WLDR = Subtest Word List-Delayed Recall of the German version of the CERAD-NP-Plus test battery; WLR = Subtest Word List-Recognition of the German version of the CERAD-NP-Plus test battery; FD = Subtest Figure Drawing of the German version of the CERAD-NP-Plus test battery; FR = Subtest Figure Recall of the German version of the CERAD-NP-Plus test battery; VF = Sum score verbal fluency (semantic and phonemic) of the German version of the CERAD-NPPlus test battery; TMT-A = Trail Making Test - Version A; TMT-B = Trail Making Test - Version B 
No other significant relationship between neuropsychological tests and abnormal slow wave activity was revealed (see Table 1). After correction for multiple comparisons, however, no significant association could be observed.

Significant effects of age on cognitive performance were found for several tests. Elderly people performed significantly worse in every neuropsychological test except for Verbal Fluency as well as Word List Recognition (for details see Table 2).

\section{Discussion}

The present study aimed at exploring age-related alterations in the distribution of generators of focal magnetic slow waves and potential associations between cognitive performance and sources of low-frequency activity in the brain.

Table 2 Influence of age on neuropsychological test performance (linear regression with constant variable age and dependent variables test results)

\begin{tabular}{lclc}
\hline $\begin{array}{l}\text { Dependent Variable } \\
\text { (neuropsychological tests) }\end{array}$ & $\beta-$ Coefficient & $F$ & $p$ \\
\hline Digit Symbol & -.67 & $F(1,51)=41.18$ & $<.001^{*}$ \\
Digit Span & -.29 & $F(1,51)=4.65$ & $.04^{*}$ \\
Mosaic Test & -.62 & $F(1,51)=32.11$ & $<.001^{*}$ \\
Benton-corr & -.64 & $F(1,51)=33.88$ & $<.001^{*}$ \\
Boston Naming Test & -.35 & $F(1,51)=7.17$ & $.01^{*}$ \\
MMSE & -.32 & $F(1,51)=5.70$ & $.02^{*}$ \\
WLL & -.50 & $F(1,51)=17.22$ & $<.001^{*}$ \\
WLDR & -.44 & $F(1,51)=11.93$ & $.001^{*}$ \\
WLR & -.21 & $F(1,51)=2.37$ & .13 \\
FD & -.31 & $F(1,51)=5.35$ & $.03^{*}$ \\
FR & -.52 & $F(1,51)=19.23$ & $<.001^{*}$ \\
VF & -.15 & $F(1,51)=1.25$ & .27 \\
TMT-A & .51 & $F(1,51)=17.88$ & $<.001^{*}$ \\
TMT-B & .55 & $F(1,51)=22.15$ & $<.001^{*}$ \\
\hline
\end{tabular}

Digit Symbol = Digit Symbol subtest of the German version of the Wechsler Adult Intelligence Scale; Digit Span = Digit Span subtest of the German version of the Wechsler Adult Intelligence Scale; Bentoncorr $=$ correct answers of the Benton Visual Retention Test (revised form); MMSE = Mini Mental State Examination; WLL = Subtest Word List-Learning of the German version of the CERAD-NP-Plus test battery; WLDR $=$ Subtest Word List-Delayed Recall of the German version of the CERAD-NP-Plus test battery; WLR $=$ Subtest Word List-Recognition of the German version of the CERAD-NP-Plus test battery; FD = Subtest Figure Drawing of the German version of the CERAD-NP-Plus test battery; FR = Subtest Figure Recall of the German version of the CERAD-NP-Plus test battery; VF = Sum score verbal fluency (semantic and phonemic) of the German version of the CERAD-NP-Plus test battery; TMT-A = Trail Making Test - Version A; TMT-B $=$ Trail Making Test - Version B

$* p<.05$
The density of focal slow wave activity in this sample of healthy adults was comparable to the younger healthy control groups of other studies (Rockstroh et al. 2007; Wienbruch 2007). A medium effect size was found for the association between age and the density of slow wave activity showing that dipole density decreases with increasing age. This effect is consistent with findings from Wienbruch (2007) who also observed a negative association between age and dipole density, albeit in a more restricted age range.

Concerning the association between dipole density and cognitive performance, no significant relationship could be found after correction for multiple comparisons. Other studies, however, suggest that focal slow wave activity can be used as a physiological marker for pathological processes in $\mathrm{MCI}$ and $\mathrm{AD}$ by Fernández and colleagues (Fernández et al. 2003, 2002; Fernandez et al. 2006). In our sample of cognitively healthy individuals, slow waves were not related to cognitive performance. Thus, in healthy aging slow wave activity declines and shows no relationship to cognitive performance. In contrast, in pathological aging, clear focal temporoparietal slow wave activity seems to be predictive of the cognitive status of the patients (Fernández et al. 2002) and is linked to the rate of atrophy in the temporal lobe (Fernández et al. 2003). This extends our understanding of focal slow wave activity suggesting that slow wave activity in the awake brain is not necessarily a marker for abnormal brain function. Slow wave activity is therefore not "abnormal" per se-it can rather be detected in all individual brains and the amount of slow wave

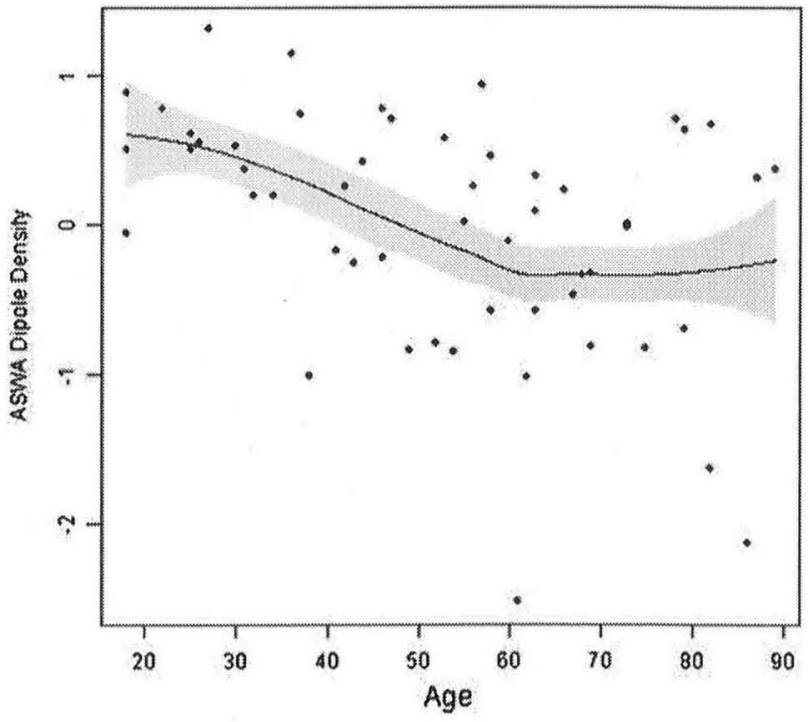

Fig. 1 Relationship between age and dipole density (z-score averaged across regions and hemispheres). The line denotes a local nonparametric kernel density smoother. The gray shading indicates pointwise $+/-$ one standard error 
activity changes over the lifetime. Therefore, we want to emphasize the importance of age-matched control groups for the detection of abnormally elevated focal slow wave activity. "Abnormal" slow waves can only be "abnormally high" in relation to a control group of the same age.

In sum, we found a general decrease of focal generators of slow wave activity during healthy aging while Morbus Alzheimer is typically associated with a focal increase in temporo-parietal brain areas. We did not observe a significant association between the extent of remaining delta dipole density and cognitive performance. Considering these results in the context of previous studies, we speculate that in neuropathological aging processes like Morbus Alzheimer or mild cognitive impairment elevated dipole density acts as a physiological marker for pathological processes whereas in healthy individuals of the same age, slow wave activity remains comparatively low and even decreases with age.

\section{References}

Ackenheil, M., Stotz, G., Dietz-Bauer, R., \& Vossen, A. I. (1999). M.I. N.I. 5.0.0. German version / DSM-IV. Munich: Psychiatrische Universitätsklinik.

Aizenstein, H. J., Nebes, R. D., Saxton, J. A., Price, J. C., Mathis, C. A., Tsopelas, N. D., et al. (2008). Frequent amyloid deposition without significant cognitive impairment among the elderly. Archives of Neurology, 65(11), 1509-1517.

Akaike, H. (1973). Information theory and an extension of the maximum likelihood principle. Paper presented at the 2nd International Symposium on Information Theory, Akademiai Kiado.

Amzica, F., \& Steriade, M. (1997). The K-complex: its slow $(<1-\mathrm{Hz})$ rhythmicity and relation to delta waves. Neurology, 49(4), 952959.

Baayen, J. C., de Jongh, A., Stam, C. J., de Munck, J. C., Jonkman, J. J., Trenite, D. G., et al. (2003). Localization of slow wave activity in patients with tumor-associated epilepsy. Brain Topography, 16 (2), 85-93.

Backman, L., Nyberg, L., Lindenberger, U., Li, S. C., \& Farde, L. (2006). The correlative triad among aging, dopamine, and cognition: current status and future prospects. Neuroscience and Biobehavioral Reviews, 30(6), 791-807.

Bartzokis, G. (2004). Age-related myelin breakdown: a developmental model of cognitive decline and Alzheimer's disease. Neurobiology of Aging, 25(1), 5-18. author reply 49-62.

Burnham, K. P., \& Anderson, D. R. (2002). Model selection and multimodel inference: A practical information-theoretic approach. Springer.

Colsher, P. L., \& Wallace, R. B. (1991). Longitudinal application of cognitive function measures in a defined population of community-dwelling elders. Annals of Epidemiology, 1(3), 215230.

Corral, M., Rodriguez, M., Amenedo, E., Sanchez, J. L., \& Diaz, F. (2006). Cognitive reserve, age, and neuropsychological performance in healthy participants. Developmental Neuropsychology, 29(3), 479-491.

de Jongh, A., de Munck, J. C., Baayen, J. C., Jonkman, E. J., Heethaar, R. M., \& van Dijk, B. W. (2001). The localization of spontaneous brain activity: first results in patients with cerebral tumors. Clinical Neurophysiology, 112(2), 378-385.

Elbert, T. (1998). Neuromagnetism. In W. Andrä \& H. Nowak (Eds.), Magnetism in medicine (pp. 190-261). London: Wiley.

Evans, D. A., Beckett, L. A., Albert, M. S., Hebert, L. E., Scherr, P A., Funkenstein, H. H., et al. (1993). Level of education and change in cognitive function in a community population of older persons. Annals of Epidemiology, 3(1), 71-77.

Fehr, T., Kissler, J., Moratti, S., Wienbruch, C., Rockstroh, B., \& Elbert, T. (2001). Source distribution of neuromagnetic slow waves and MEG-Delta activity in Schizophrenic patients. Biological Psychiatry, 49, 1-10.

Fehr, T., Kissler, J., Wienbruch, C., Moratti, S., Elbert, T., Watzl, H., et al. (2003). Source distribution of neuromagnetic slow-wave activity in schizophrenic patients - effects of activation. Schizophrenia Research, 63, 63-71.

Fernández, A., Maestú, F., Amo, C., Gil, P., Fehr, T., Wienbruch, C., et al. (2002). Focal temporoparietal slow activity in Alzheimer's Disease revealed by magnetencephalography. Biological Psychiatry, $52,764-770$.

Fernández, A., Arrazola, J., Maestu, F., Amo, C., Gil-Gregorio, P., Wienbruch, C., et al. (2003). Correlations of hippocampal atrophy and focal low-frequency magnetic activity in Alzheimer disease: volumetric MR imaging-magnetoencephalographic study. AJNR. American Journal of Neuroradiology, 24(3), 481487.

Fernández, A., Rodriguez-Palancas, A., López-Ibor, M., Zuluaga, P., Turrero, A., Maestú, F., et al. (2005). Increased occipital delta dipole density in major depressive disorder determined by magnetoencephalography. Journal of Psychiatry \& Neuroscience, $30(1), 17-23$.

Fernández, A., Hornero, R., Mayo, A., Poza, J., Gil-Gregorio, P., \& Ortiz, T. (2006). MEG spectral profile in Alzheimer's disease and mild cognitive impairment. Clinical Neurophysiology, $117(2)$, 306-314.

Fernandez, A., Turrero, A., Zuluaga, P., Gil, P., Maestu, F., Campo, P., et al. (2006). Magnetoencephalographic parietal delta dipole density in mild cognitive impairment: preliminary results of a method to estimate the risk of developing Alzheimer disease. Archives of Neurology, 63(3), 427-430.

Galluzzi, S., Beltramello, A., Filippi, M., \& Frisoni, G. B. (2008). Aging. Neurological Sciences, 29 (Suppl 3), 296-300.

Giedd, J. N., Blumenthal, J., Jeffries, N. O., Castellanos, F. X., Liu, H., Zijdenbos, A., et al. (1999). Brain development during childhood and adolescence: a longitudinal MRI study. Nature Neuroscience, 2(10), 861-863.

Gloor, P., Ball, G., \& Schaul, N. (1977). Brain lesions that produce delta waves in the EEG. Neurology, 27(4), 326-333.

Good, C. D., Johnsrude, I. S., Ashburner, J., Henson, R. N., Friston, K. J., \& Frackowiak, R. S. (2001). A voxel-based morphometric study of ageing in 465 normal adult human brains. Neurolmage, 14(1 Pt 1), 21-36.

Kolassa, I. T., Wienbruch, C., Neuner, F., Schauer, M., Ruf, M., Odenwald, M., et al. (2007). Altered oscillatory brain dynamics after repeated traumatic stress. BMC Psychiatry, 7, 56.

Lindenberger, U., \& Reischies, F. M. (1999). Limits and potentials of intellectual functioning in old age. In P. B. Baltes \& K. U. Mayer (Eds.), The Berlin aging study: Aging from 70 to 100. Berlin: Akademie Verlag.

Meinzer, M., Elbert, T., Wienbruch, C., Djundja, D., Barthel, G., \& Rockstroh, B. (2004). Intensive language training enhances brain plasticity in chronic aphasia. BMC Biology, 2, 20.

Mintun, M. A., Larossa, G. N., Sheline, Y. I., Dence, C. S., Lee, S. Y., Mach, R. H., et al. (2006). [11C]PIB in a nondemented population: potential antecedent marker of Alzheimer disease. Neurology, 67(3), 446-452. 
Morris, J. C., Mohs, R. C., Rogers, H., Fillenbaum, G., \& Heyman, A. (1988). Consortium to establish a registry for Alzheimer's disease (CERAD) clinical and neuropsychological assessment of Alzheimer's disease. Psychopharmacology Bulletin, 24(4), 641-652.

Niedermeyer, E., \& Lopes da Silva, F. (1987). Electroencephalography: Basic principles, clinical applications and related fields. Baltimore: Urban \& Schwarzenberg.

Oldfield, R. (1971). The assessment and analysis of handedness: the Edinburgh inventory. Neuropsychologia, 9(1), 97-113.

Pike, K. E., Savage, G., Villemagne, V. L., Ng, S., Moss, S. A., Maruff, P., et al. (2007). Beta-amyloid imaging and memory in non-demented individuals: evidence for preclinical Alzheimer's disease. Brain, 130(Pt 11), 2837-2844.

Raz, N., Lindenberger, U., Rodrigue, K. M., Kennedy, K. M., Head, D., Williamson, A., et al. (2005). Regional brain changes in aging healthy adults: general trends, individual differences and modifiers. Cerebral Cortex, 15(11), 1676-1689.

Rockstroh, B. S., Wienbruch, C., Ray, W. J., \& Elbert, T. (2007). Abnormal oscillatory brain dynamics in schizophrenia: a sign of deviant communication in neural network? BMC Psychiatry, 7, 44.

Salthouse, T. A. (2006). Mental exercise and mental aging: evaluating the validity of the "Use It or Lose It" hypothesis. Perspectives on Psychological Science, 1, 68-87.
Steck, P. H. (2005). A revision of A. L. Benton's Visual Retention Test (BVRT) in two parallel forms. Archives of Clinical Neuropsychology, 20, 409-416.

Team, R. D. C. (2008). R: A language and environment for statistical computing (version 2.8.1). Vienna, Austria: R Foundation for Statisitcal Computing.

Terry, R. D., \& Katzman, R. (2001). Life span and synapses: will there be a primary senile dementia? Neurobiology of Aging, 22(3), 347-348. discussion 353-344.

Tewes, U. (1991). Hamburg-Wechsler-Intelligenztest für Erwachsene (HAWIE-R). Bern: Verlag Hans Huber.

Vieth, J. B., Kober, H., \& Grummich, P. (1996). Sources of spontaneous slow waves associated with brain lesions, localized by using the MEG. Brain Topography, 8(3), 215-221.

Wienbruch, C. (2007). Abnormal slow wave mapping (ASWAM)-A tool for the investigation of abnormal slow wave activity in the human brain. Journal of Neuroscience Methods, 163(1), 119-127.

Wienbruch, C., Moratti, S., Elbert, T., Vogel, U., Fehr, T., Kissler, J., et al. (2003). Source distribution of neuromagnetic slow wave activity in schizophrenic and depressive patients. Clinical Neurophysiology, 114, 2052-2060.

Wilson, R. S., Beckett, L. A., Bennett, D. A., Albert, M. S., \& Evans, D. A. (1999). Change in cognitive function in older persons from a community population: relation to age and Alzheimer disease. Archives of Neurlogy, 56(10), 1274-1279. 